\title{
Complete response of advanced hepatocellular carcinoma to sorafenib: another case and a comprehensive review
}

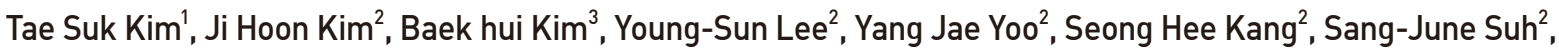 \\ Young Kul Jung ${ }^{2}$, Yeon Seok Seo ${ }^{2}$, Hyung Joon Yim², Jong Eun Yeon ${ }^{2}$, and Kwan Soo Byun ${ }^{2}$ \\ 'Department of Internal Medicine, Kangwon National University School of Medicine, Gangwon; ${ }^{2}$ Department of Internal Medicine, Korea \\ University College of Medicine, Seoul; ${ }^{3}$ Department of Pathology, Korea University College of Medicine, Seoul, Korea
}

\begin{abstract}
Since sorafenib was introduced in 2007 for treating advanced hepatocellular carcinoma (HCC), 15 patients have achieved a complete response (CR) in advanced HCC. However, only four of these reports can be regarded as real CRs involving adequate assessments including imaging, serum tumor markers, and histologic examinations of completely resected specimens. A 54-year-old man with hepatitis C virus (HCV)-related liver cirrhosis (LC) presented to our unit. A CT scan demonstrated a 3.8-cm arterial hypervascular/portal-washout mass in the right lobe and invasion in the right portal vein. Twelve weeks after beginning sorafenib therapy, the AFP level was normalized and a CT scan showed a prominent decrease in the hepatic mass and a significant decrease in the volume of portal vein thrombosis (PVT). The patient received a right liver hemihepatectomy after 12 months. No viable tumor cells were found in the resected specimen, and there was no thrombotic obstruction of the portal vein. Twelve months later the patient showed no clinical evidence of HCC recurrence. This is the first case of CR in HCC treatment following sorafenib with histologically confirmed HCVrelated HCC without LC evidence, HCC with PVT, and a follow-up of longer than 12 months. This case seems to be an extremely unusual clinical outcome in advanced HCC. (Clin Mol Hepatol 2017;23:340-346)
\end{abstract}

Keywords: Hepatocellular carcinoma; Sorafenib; Response evaluation criteria in solid tumors; Hepatitis C

\section{INTRODUCTION}

Hepatocelluar carcinoma (HCC) is the fifth most frequent type of malignancy worldwide and is the second most common cause of cancer-related death in the world. Only 30-40\% patients with HCC are eligible for potentially curative treatments, such as liver transplantation, surgical resection, radiofrequency ablation (RFA) or percutaneous ethanol injection (PEI). A substantial number of
HCC cases are diagnosed at advanced stages, and the median survival following diagnosis is approximately 6 to 20 months.' Therapeutic options for patients with late stage HCC at the time of diagnosis include transarterial chemoembolization (TACE) and/ or systemic chemotherapy such as sorafenib. Sorafenib (Nexavar ${ }^{\circledR}$ ) is a small molecule that inhibits tumor-cell proliferation and angiogenesis and represents the sole chemotherapeutic agent with proven survival benefit in HCC. Following a successful

\section{Abbreviations:}

AFP, alpha-fetoprotein; ALT, alanine aminotransferase; BCLC, Barcelona Liver Cancer Clinic; $\mathrm{CR}$, complete response; $\mathrm{HCC}$, hepatocellular carcinoma; $\mathrm{HCV}$, hepatitis $\mathrm{C}$ virus; HFSR, hand-foot skin reaction; MRI, magnetic resonance imaging; PEl, percutaneous ethanol injection; PVT, portal vein thrombosis; RFA, radiofrequency ablation; TACE, transarterial chemoembolization

\section{Corresponding author : Ji Hoon Kim}

Department of Internal Medicine, Korea University College of Medicine, 148 Gurodong-ro, Guro-gu, Seoul 08308, Korea

Tel: +82-2-2626-3011, Fax: +82-2626-2024

E-mail:kjhhepar@naver.com

http://orcid.org/0000-0003-3924-0434 
phase III pivotal trial ${ }^{2}$, it has been widely approved around the world for the treatment of advanced HCC. However, sorafenib has not yet been considered as a cytoreductive agents, because the complete response $(C R)$ rate was $0 \%$ and partial response rate was only $2.2 \%$ (10/449) in two pivotal phase III trial. ${ }^{2,3}$ In fact, the objective response rate remains infrequent and $C R$ is extremely rare in real-life practice. Because complete resection is the mainstay for HCC treatment and the only curable option, it is important to investigate methods to improve the resectability of HCC using neoadjuvant therapy. Since 2008, a total of fifteen cases of $C R$ were reported. Moreover, only four cases of CR were confirmed histologically through follow-up surgical resection and only one of these were associated with underlying hepatitis $C$ virus infection with only case having the longest (6 months) recurrence free follow up duration. We present a case of a 54 year old male with underlying hepatitis C virus (HCV) infection who experienced complete histologic remission of his HCC following sorafenib treatment without recurrence after 12 months follow up.

\section{CASE REPORT}

A 54-year-old man with HCV (genotype 2a) related liver cirrhosis (LC) presented to our unit due to diffuse abdominal pain. A computed tomography (CT) scan and magnetic resonance imaging(MRI) revealed a $3.8 \mathrm{~cm}$, arterial hypervascular/portal washout mass in the right lobe with invasion of the right portal vein (Fig. 1). Serum alpha-fetoprotein (AFP) level was $12,463 \mathrm{ng} /$ $\mathrm{mL}$. Typical radiographic features with a marked elevation of AFP in HCV-related LC led to the diagnosis of HCC without performing liver biopsy. Further laboratory findings included alanine amino- transferase (ALT) $47 \mathrm{IU} / \mathrm{L}$, total bilirubin $0.49 \mathrm{mg} / \mathrm{dL}$, albumin 3.9 g/dL, PT INR 0.96 and HCV RNA 89,466 IU/mL. The patient had no encephalopathy or ascites. His liver function was well compensated with Child-Pugh A. The patient's Eastern Cooperative Oncology Group performance status was 1.

The patient's HCC stage based on the Barcelona Liver Cancer Clinic (BCLC) was advanced stage (BCLC C). Therefore, he underwent treatment with sorafenib. Sorafenib therapy was initiated at $400 \mathrm{mg}$ orally, twice daily dosing. The patient tolerated the therapy well with minimal toxicity but did experience grade 2 handfoot skin reaction (HFSR), which was well controlled with ointment and topical analgesics and was not dose limiting. Twelve weeks after beginning therapy, the AFP level was normalized (3.8 $\mathrm{ng} / \mathrm{mL}$ ). In addition, follow-up CT scan showed prominent decrease in hepatic mass with central necrotic changes without any enhancing areas as well as a significant decrease in volume of the right portal vein thrombosis (Fig. 2A, 2B). The tumor response to sorafenib was assessed as complete response by the modified response evaluation criteria in solid tumors criteria. ${ }^{4}$ We continued sorafenib therapy to eradicate potentially microscopic tumor cell remnants. After 12 months of sorafenib therapy, the non-enhancing hypodense lesion decreased in size and there was no visible tumor thrombosis in the right portal vein (Fig. 2C, 2D). The patient then underwent right hemihepatectomy with complete resection of the residual tumor.

Histologic evaluation of the resected hemihepatectomy specimen revealed macrophages and inflammatory cells in a background of fibrinoid necrosis with no viable tumor cells. In the hepatic parenchyma surrounding the mass, chronic hepatitis with fibrosis was observed. There were no viable tumor cells in the thrombosis of the portal vein. Of interest, thrombotic materia
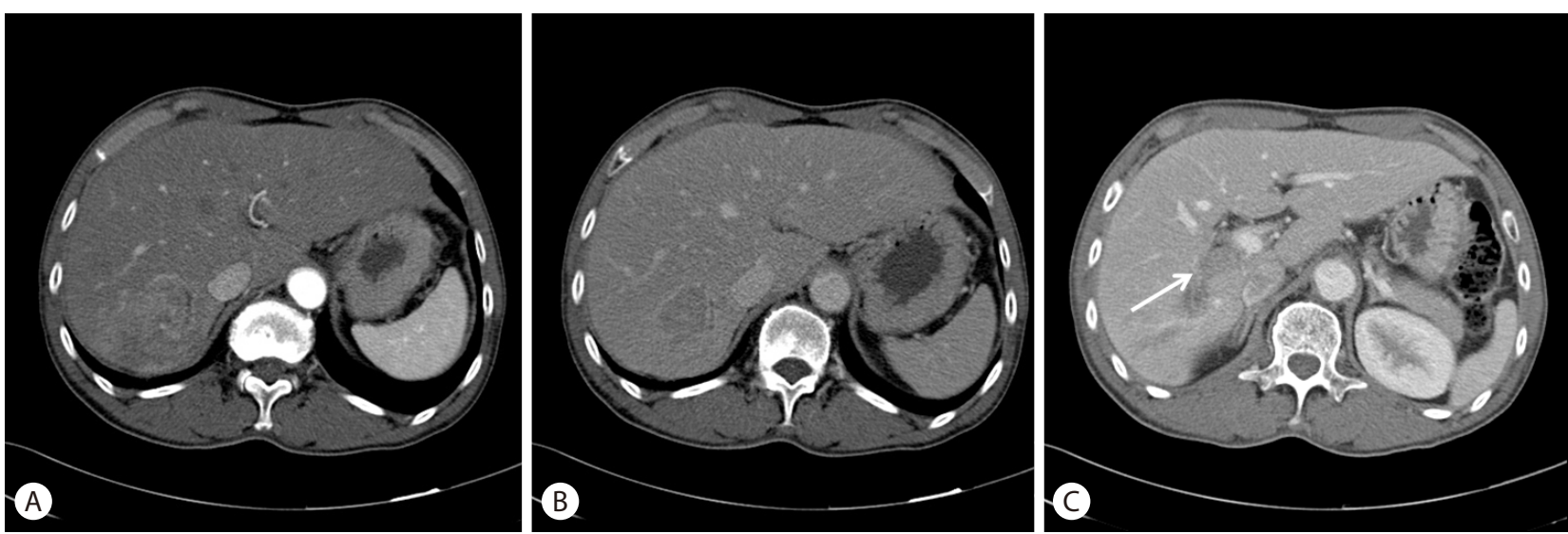

Figure 1. Baseline CT scan showed $3.8 \mathrm{~cm}$ sized arterial heterogenously enhancing (A) and delayed wash out (B) hepatic mass. CT scan of portal phase (C) showed right portal vein invasion (arrow) 


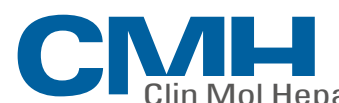

Clin Mol Hepatol

Volume 23 Number 4 December 2017

showed microscopic features similar with the mass lesion (Fig. 3). Because there were no viable tumor cells in the total 14 sections, immunohistochemical staining was not performed. Twelve months after the resection, the patient showed no clinical evidence of recurrence of HCC.

\section{DISCUSSION}

Systemic chemotherapy has not been used primary for patients with advanced HCC because HCC has been considered a relatively chemotherapy resistant tumor. HCC has a high rate of expression of drug resistance genes, such as the heat shock proteins, pglycoprotein, glutathione-S-transferase, and mutations in p53. Many molecular pathways contribute to HCC carcinogenesis. In addition, outcomes after chemotherapy are significantly influ- enced by underlying liver dysfunction. Before the sorafenib era, doxorubicin was the mainstay of therapy for advanced HCC refractory to locoregional therapy with approximate response rates of $15-20 \% .{ }^{5} 5$-fluorouracil, epirubicin, cisplatin, etoposide, doxorubicin, and their combinations were used in treatment for advanced HCC and the response rate of previous studies have been $13-39 \%{ }^{6}$ However, prior to sorafenib, systemic chemotherapy for HCC did not show significant survival benefit compared to placebo in large controlled study. Sorafenib is the first multi kinase inhibitor to be approved by the United States Food and Drug Administration for the treatment of advanced HCC as of 2007. Sorafenib simultaneously inhibits molecular components of the mitogen-activated protein kinase/extracellular-signal-regulated kinase (RAF/MEK/ERK) signaling pathway and platelet-derived growth factor receptor- $\beta$ /vascular endothelial growth factor receptor (VEGFR) and thereby inhibit tumor growth and inhibit an-
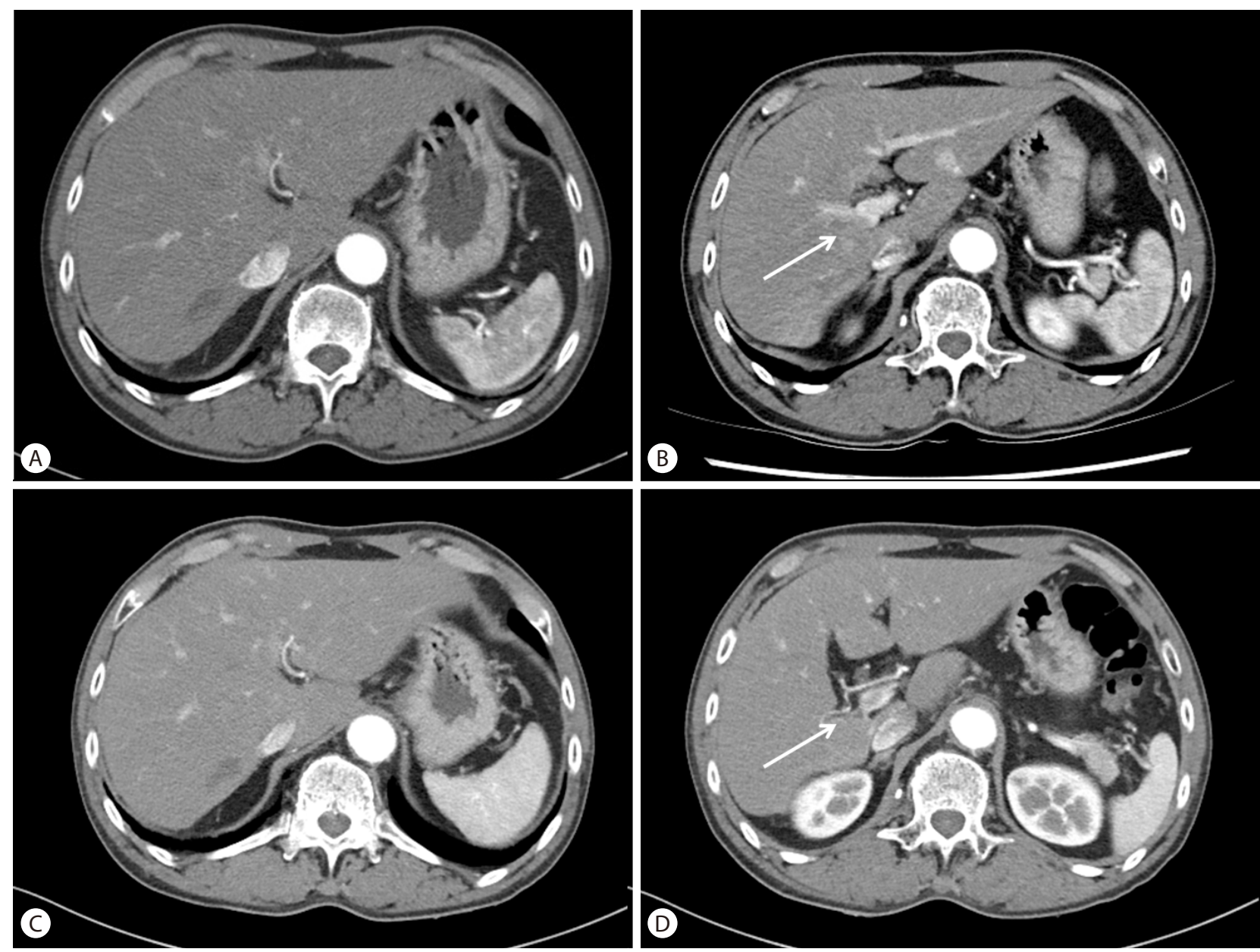

Figure 2. Follow up CT scan at 12 weeks after sorafenib Therapy showed significantly decreased mass lesion (A) and portal vein invasion (B, arrow) without arterial enhancement. Follow up CT scan at 12 months after sorafenib Therapy showed more contracted mass lesion (C) and more obliterated portal vein ( $\mathrm{D}$, arrow). 

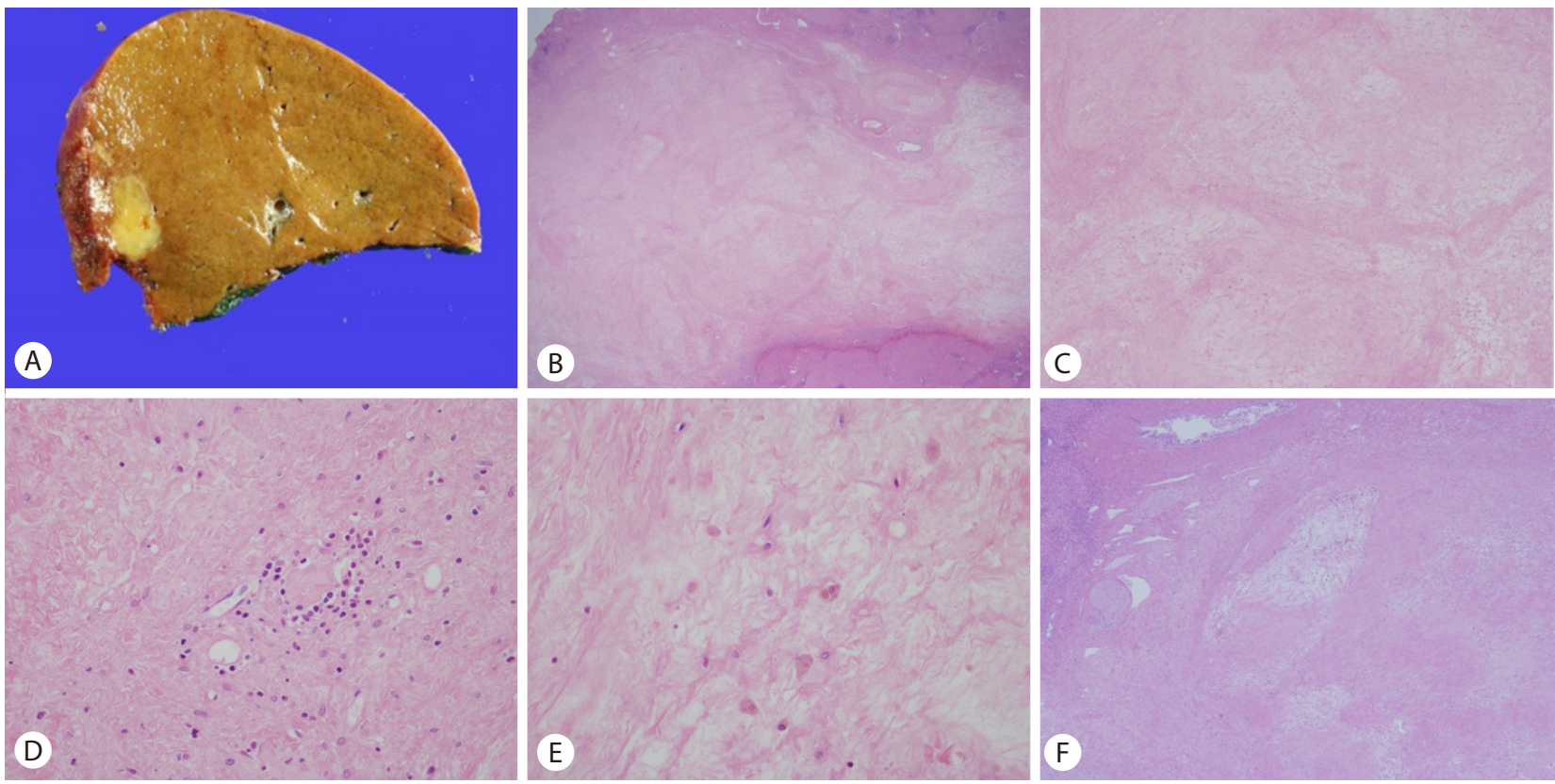

Figure 3. Gross photo and microscopic features of resected hepatic lesion. (A) Well circumscribed, soft, yellow and lobulated HCC in a background of non-cirrhotic liver. (B) Scanning power view shows fibrinoid necrosis. In the hepatic parenchyma around mass, chronic hepatitis with fibrosis is noted (original magnification $\times 12.5$ ). (C) No viable tumor cell is observed in the lower power view of the lesion (original magnification $\times 40)$. (D, E) Scattered macrophages, Lymphocytes and plasma cells are seen in the fibrinoid necrotic background. Original magnification (D) $\times 200$ and (E) $\times 400$. (F) Low power view shows vascular wall and thrombotic material of portal vein. Thrombotic material shows features similar with the mass lesion. Some macrophages and inflammatory cells with no viable tumor cell in the fibrinoid necrotic material (original magnification ×40).

giogenesis. ${ }^{7}$ In the first randomized controlled study, sorafenib treatment was found to reduce the risk of death after one year by $31 \%$ and to prolong median survival by approximately three months compared to placebo. ${ }^{2}$ Since these discoveries, sorafenib has been regarded as the primary therapeutic strategy for advanced HCC.

However, one of the major drawbacks of sorafenib treatment is the low rate of objective response. Complete surgical resection is the only potentially curative therapy. Therefore, the goal of sorafenib introduction was not only to increase survival time but also to decrease tumor size and the extent to which is resectable for complete elimination of residual lesions. Disappointingly, in two pivotal multicenter phase III placebo-controlled clinical trials of sorafenib for advanced HCC ${ }^{2,3}$, no CR was reported in 449 patients who were randomly assigned to sorafenib and only $2 \%$ and $3.3 \%$ patients were considered as partial responders according to RECIST criteria.

Since the advent of sorafenib for the treatment of advanced HCC, a total of fourteen reports have documented fifteen patients who achieved CR in advanced HCC. However, their clinical findings and courses have been heterogenous (Table 1). The patients have harbored a variety of underlying liver diseases; six with $\mathrm{HCV}$, four patients with hepatitis B virus (HBV), two with alcoholic cirrhosis, two with hereditary hemochromatosis (HHC), and one HBV with human immunodeficiency virus. Most of the patients were male gender, and one Japanese female patient, who had achieved CR with underlying $\mathrm{HCV}^{8}{ }^{8}$ Among all cases, five HCC cases were confined to the liver, seven cases were intrahepatic HCC with metastasis, and three cases were present in the form of metastatic lesions without an intrahepatic lesion. The fifteen cases of $C R$ included nine cases with oral sorafenib treatment only and six with locoregional treatment or systemic chemotherapy prior to treatment with sorafenib. At the time of diagnosis, four patients were found to have low AFP levels ( $<400$ $\mathrm{ng} / \mathrm{mL}$ ). Among these, two cases had no intrahepatic lesion with metastatic lesions only. Moreover, eleven of the $C R$ cases were evaluated by only radiologic methods, with only four documents of $C R$ supported by imaging, serum tumor marker assessment, and histological examination. Sorafenib treatment time to cessation was 0.4 months up to 22 months. CR was achieved in six months in five cases.

Although a total of fifteen cases of $C R$ have been documented worldwide, only four documents were regarded as true $C R$ with adequate assessment methods including imaging, serum tumor 


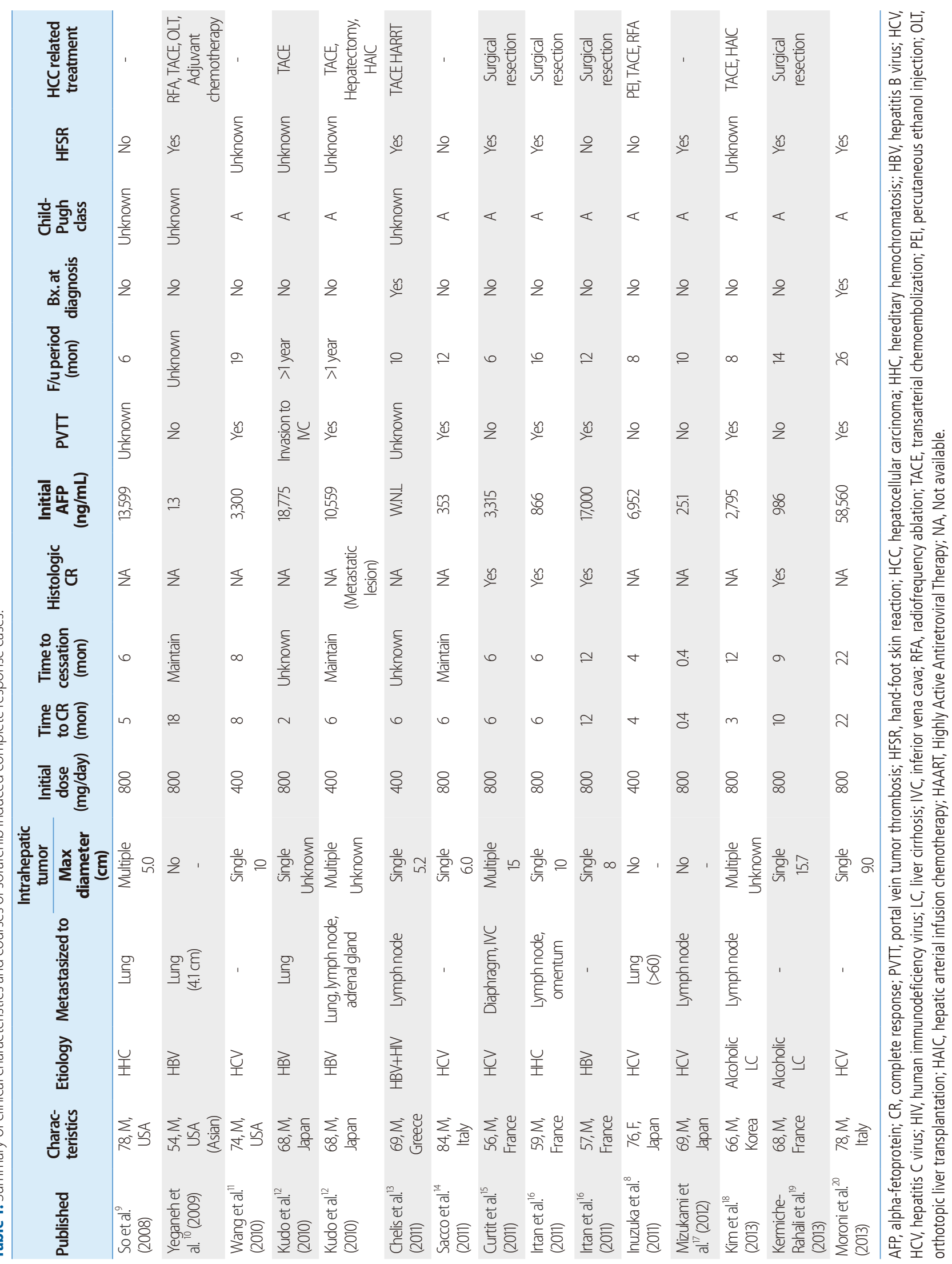


Tae Suk Kim, et al.

CR of advanced HCC to sorafenib

marker, and histologic examination of completely resected specimen. Two of these had documented portal vein thrombosis, which is one of main negative prognostic factors of HCC. There were various underlying etiologies and progression of underlying liver disease, including advanced fibrosis (Metavir 3) with HBV, LC with HCV, Alcoholic LC, and advanced fibrosis (Metavir 3) with HHC. Generally, recurrence 2 year after resection has been regarded as de novo, but recurrence within 1-2 year after resection could be accepted as de novo recurrence. Among these cases, the reported follow up duration of two cases were 16 and 6 months, respectively. One HCV-related HCC case with LC did not have portal vein thrombosis. The present report is first case of $C R$ through sorafenib containing histological confirmed, HCV-related HCC without histologic evidence of hepatic cirrhosis, HCC with portal vein thrombosis, and more than 12 months of follow up duration.

In conclusion, this case demonstrates an extremely unusual clinical outcome in advanced HCC. Moreover, the reaction mechanism by which complete histologic response is was achieved remains unclear. Complete histologic response could be demonstrated in rare cases and demonstrates that sorafenib therapy could be a bridge to complete resection in anticipation of a cure for $\mathrm{HCC}$

\section{Conflicts of Interest}

The authors have no conflicts to disclose.

\section{REFERENCES}

1. A new prognostic system for hepatocellular carcinoma: a retrospective study of 435 patients: the Cancer of the Liver Italian Program (CLIP) investigators. Hepatology 1998;28:751-755.

2. Llovet JM, Ricci S, Mazzaferro V, Hilgard P, Gane E, Blanc JF, et al. Sorafenib in advanced hepatocellular carcinoma. N Engl J Med 2008;359:378-390.

3. Cheng AL, Kang YK, Chen Z, Tsao CJ, Qin S, Kim JS, et al. Efficacy and safety of sorafenib in patients in the Asia-Pacific region with advanced hepatocellular carcinoma: a phase III randomised, doubleblind, placebo-controlled trial. Lancet Oncol 2009;10:25-34.

4. Lencioni R, Llovet JM. Modified RECIST (mRECIST) assessment for hepatocellular carcinoma. Semin Liver Dis 2010;30:52-60.

5. Yeo W, Mok TS, Zee B, Leung TW, Lai PB, Lau WY, et al. A randomized phase III study of doxorubicin versus cisplatin/interferon alpha2b/doxorubicin/fluorouracil (PIAF) combination chemotherapy for unresectable hepatocellular carcinoma. J Natl Cancer Inst
2005;97:1532-1538.

6. Zhu AX. Systemic therapy of advanced hepatocellular carcinoma: how hopeful should we be? Oncologist 2006;11:790-800.

7. Wilhelm SM, Adnane L, Newell P, Villanueva A, Llovet JM, Lynch M. Preclinical overview of sorafenib, a multikinase inhibitor that targets both Raf and VEGF and PDGF receptor tyrosine kinase signaling. Mol Cancer Ther 2008;7:3129-3140.

8. Inuzuka T, Nishikawa H, Sekikawa A, Takeda H, Henmi S, Sakamoto $A$, et al. Complete response of advanced hepatocellular carcinoma with multiple lung metastases treated with sorafenib: a case report. Oncology 2011;81(Suppl):152-157.

9. So BJ, Bekaii-Saab T, Bloomston MA, Patel T. Complete clinical response of metastatic hepatocellular carcinoma to sorafenib in a patient with hemochromatosis: a case report. J Hematol Oncol 2008;1:18

10. Yeganeh M, Finn RS, Saab S. Apparent remission of a solitary metastatic pulmonary lesion in a liver transplant recipient treated with sorafenib. Am J Transplant 2009;9:2851-2854.

11. Wang SX, Byrnes A, Verma S, Pancoast JR, Rixe O. Complete remission of unresectable hepatocellular carcinoma treated with reduced dose of sorafenib: a case report. Target Oncol 2010;5:59-63.

12. Kudo M, Ueshima K. Positioning of a molecular-targeted agent, sorafenib, in the treatment algorithm for hepatocellular carcinoma and implication of many complete remission cases in Japan. Oncology 2010;78:154-166.

13. Chelis L, Ntinos N, Souftas V, Deftereos S, Xenidis N, Chamalidou E, et al. Complete response after sorafenib therapy for hepatocellular carcinoma in an HIV-HBV co infected patient: Possible synergy with HAART? A case report. Med Oncol 2011;28(Suppl 1):S165-S168.

14. Sacco R, Bargellini I, Gianluigi G, Bertini M, Bozzi E, Altomare E, et al. Complete response for advanced liver cancer during sorafenib therapy: case report. BMC gastroenterol 2011;11:4.

15. Curtit E, Thiery-Vuillemin A, Nguyen T, Heyd B, Pivot X, Di Martino $V$, et al. Complete histologic response induced by sorafenib in advanced hepatocellular carcinoma: a case report. J Clin Oncol 2011;29:e330-e332.

16. Irtan S, Chopin-Laly X, Ronot M, Faivre S, Paradis V, Belghiti J. Complete regression of locally advanced hepatocellular carcinoma induced by sorafenib allowing curative resection. Liver Int 2011;31:740-743.

17. Mizukami H, Kagawa T, Arase Y, Nakahara F, Tsuruya K, Anzai K, et al. Complete response after short-term sorafenib treatment in a patient with lymph node metastasis of hepatocellular carcinoma. Case Rep Oncol 2012;5:380-384.

18. Kim MS, Jin YJ, Lee JW, Lee JI, Kim YS, Lee SY, et al. Complete remission of advanced hepatocellular carcinoma by sorafenib: A case report. World J Gastrointest Oncol 2013;5:38-42.

19. Kermiche-Rahali S, Di Fiore A, Drieux F, Di Fiore F, François A, Scotté 
M. Complete pathological regression of hepatocellular carcinoma with portal vein thrombosis treated with sorafenib. World J Surg Oncol 2013;11:171.
20. Moroni M, Zanlorenzi L. Complete regression following sorafenib in unresectable, locally advanced hepatocellular carcinoma. Future Oncol 2013;9:1231-1237. 